\title{
A Constructive Genetic Algorithm for Gate Matrix Layout Problems
}

\begin{tabular}{|c|c|}
\hline Alexandre César Muniz de Oliveira & Luiz Antonio Nogueira Lorena \\
\hline $\begin{array}{c}\text { DEINF - Universidade Federal do Maranhão } \\
\text { Campus Bacanga, São Luís, MA, Brasil } \\
\text { acmo@ deinf.ufma.br }\end{array}$ & LAC - Instituto Nacional de Pesquisas Espaciais \\
& Jd da Granja, 12201-970, S. J. dos Campos, SP, Brasil \\
& $\underline{\text { lorena@ lac.inpe.br }}$ \\
\hline
\end{tabular}

Abstract - This paper describes an application of a Constructive Genetic Algorithm $(C G A)$ to the Gate Matrix Layout Problem (GMLP). The GMLP happens in very large scale integration (VLSI) design, and can be described as a problem of assigning a set of circuit nodes (gates) in an optimal sequence, such that the layout area is minimized, as a consequence of optimizing the number of tracks necessary to cover the gates interconnection. The $C G A$ has a number of new features compared to a traditional genetic algorithm. These include a population of dynamic size composed of schemata and structures, and the possibility of using heuristics in structure representation and in the fitness function definitions. The application of $C G A$ to $G M L P$ uses a 2-Opt like heuristic to define the fitness functions and the mutation operator. Computational tests are presented using available instances taken from the literature.

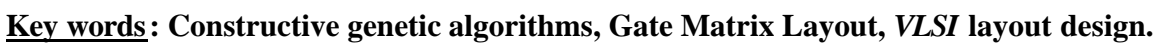

\section{INTRODUCTION}

Gate Matrix Layout problems (GMLP) are related to one-dimensional logic arrays and programmable logic arrays folding [1,2]. In very large scale integration design (VLSI design), the goal is to arrange a set of circuit nodes (gates) in an optimal sequence, such that the layout area is minimized, e.g., it minimizes the number of tracks necessary to cover the gates interconnection.

Fig. 1 shows an example of gate matrix, where the gates are numbered from 1 to 9 and the dots are the connection requests (Fig.1a). A group of gates at the same connection is called net. There are 7 nets in circuit of Fig.1. To connect gates 1,3,4 and 7 of the net 1, it is necessary to cross gates that are not part of this net. Moreover, non-overlapping nets can be placed at the same connection track. To compute the number of tracks to cover all nets, it is enough to verify the maximum of overlapping nets. The number of tracks is an important cost factor of VLSI circuits. 


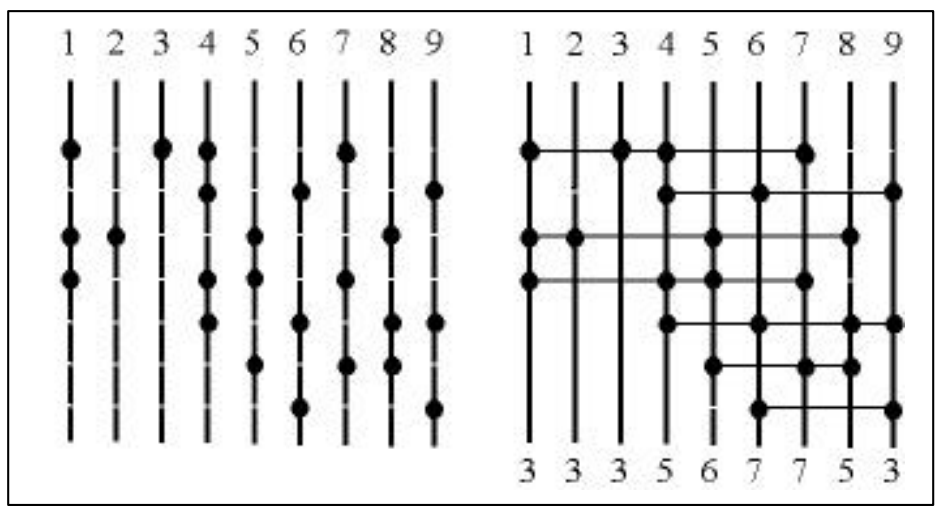

Fig.1 - Gate matrix. a) Original matrix; b) Gate matrix derived by interconnection of gates at same net

Fig. $1 \mathrm{~b}$ shows, at the bottom, the number of overlaps at each gate. A typical instance of the GMLP is composed of an $I x J$ binary matrix, where $I$ and $J$ are the numbers of nets and gates, respectively. The original matrix is represented in the following by a matrix $a$ where all dots are transformed in ones and the other positions receive zeros, and the derived interconnected matrix will be denoted by $b$, which is generated by transforming in ones all zeros between leftmost and rightmost ones in matrix a. Fig. 2 shows the matrices $a$ and $b$ related to the matrices in Fig.1.

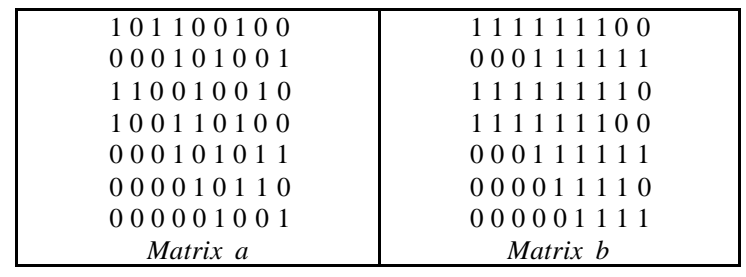

Fig. 2 - Derived matrices $a$ and $b$.

The number of tracks $(T R)$ is easily calculated by:

$$
T R=\max _{j \in\{1, \ldots, J\}} \sum_{i=1}^{I} b_{i j}=\max \{3,3,3,5,6,7,7,5,3\}=7
$$

Besides, there is also the cost of connection of the gates that symbolizes the amount of necessary metal to cover the nets. In this example, the total wire length $(W L)$ is:

$$
W L=\sum_{j=1}^{J} \sum_{i=1}^{I} b_{i j}-I=(3+3+3+5+6+7+7+5+3)-7=35
$$

$G M L P$ is also a permutation problem, and considering that the initial sequence of gates is $\{1,2,3,4, \ldots, \mathrm{J}\}$, both $T R$ and $W L$ can be improved by permutated matrices that represent particular sequences of gates. 
Fig. 3 shows a permutated gate matrix corresponding to the sequence of gates $\{6,9,4,8,7,5,1,2,3\}$. The $T R$ is reduced to 5 and the new $W L$ is 22 . Nets 3 and 7 can be placed at the same connection track (the same for nets 2 and 6).

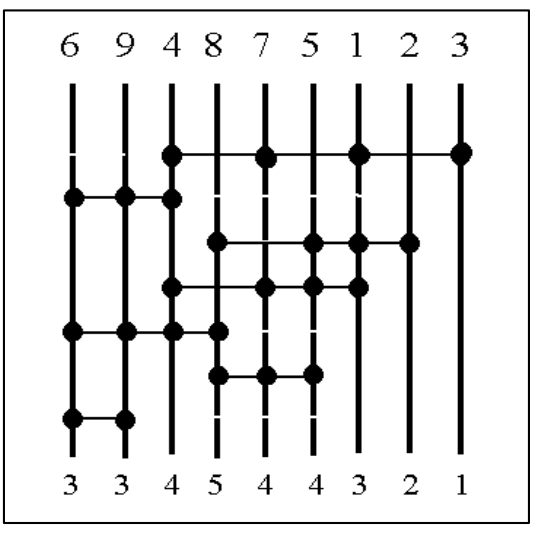

Fig. 3 - Permutated (optimal) solution

This paper describes the application of a Genetic Algorithm (GA) to problem GMLP. Traditional GAs work on a set of variables called structures. For applying them to optimization problems, the first step is the definition of a coding scheme that allows a one-to-one mapping between solutions and structures. A fitness function assigns a numeric value to each member of the current population (a collection of structures). The genetic operators used are selection (like tournament or biased roulette wheel) working together with a number of crossover and mutation operators. The best structure is kept after a predefined number of generations [3-5].

Holland put forward the "building block" hypothesis (schema formation and conservation) as a theoretical basis for the GA mechanism [4]. In his view, avoiding disruption of good schema is the basis for the good behavior of a GA. Schemata can be defined as parts of the structures, where some data is missing, and a major problem is that they are evaluated indirectly, via evaluation of their instances (structures). Goldberg and collaborators have addressed the problem of schemata evaluation and introduced the messy-GA [6-8]. In a different approach, Lorena and Furtado [9] proposed recently the Constructive Genetic Algorithm $(C G A)$ as an alternative to a traditional $G A$ approaches, particularly, for evaluating schemata directly [9].

The $C G A$ evolves a population initially formed only by schemata, controlled by recombination, to a population of well-adapted structures (schemata instantiation) and schemata. It was applied to clustering 
problems [9], location problems and timetabling problems [10]. The GMLP is the first permutation like problem modeled and solved using the $C G A$ approach.

The $C G A$ application can be divided in two phases, the constructive and the optimal:

- The constructive phase is used to build a population of quality solutions, composed of well-adapted schemata and structures, through operators as selection, recombination and specific heuristics.

- The optimal phase is conducted simultaneously and transforms the optimization objectives of the original problem on an interval minimization problem that evaluates schemata and structures in a common way.

This paper is organized as follows. Section 2 presents the aspects of modeling for schema and structure representations and the consideration of the $G M L P$ as a bi-objective optimization problem. Section 3 describes the $C G A$ operators, namely, selection, recombination and mutation, as well as a $C G A$ pseudocode. Section 4 shows computational results using instances taken from the literature.

II. CGA MODELING

In this section the modeling phase of the $C G A$ is described. The GMLP is formulated as a bi-objective optimization problem. To attain the objective of evaluating schemata and structures in a common way, two fitness functions are defined on the space of all schemata and structures that can be obtained using a specific representation. An evolution process will be described in the next section. This process considers the two objectives on an adaptive rejection threshold, which gives ranks to individuals in population and yields a dynamic population.

Very simple structure and schema representations are implemented to the GMLP. A direct alphabet of symbols (natural numbers) represents the gate sequence and each gate is associated to a column of binary numbers, representing the connection request with each net. The 1 to $\mathrm{J}$ values are reserved to gate number and the symbol \# is used to express indetermination (\# - do not care) on schemata.

Fig.4 shows the representation for the GMLP instance of Fig.1 and a permutation over it, representing structures and an example of schema. 


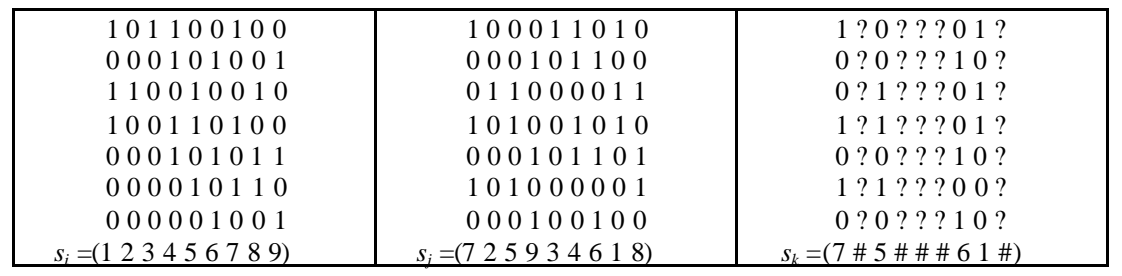

Fig.4 - a) Net-gate requirements matrix and structure $s_{i}($ Fig.2a); b) Example of permutated matrix and corresponding structure $s_{j}$; c) Example of schema.

Let $X$ be the space of all schemata and structures that can be created by this representation. The GMLP is modeled as the following Bi-objective Optimization Problem (BOP):

$$
\begin{array}{lc}
\text { Min } & \left\{g\left(s_{k}\right)-f\left(s_{k}\right)\right\} \\
\text { Max } & g\left(s_{k}\right) \\
\text { subject to } & g\left(s_{k}\right) \geq f\left(s_{k}\right) \\
& \forall s_{k} \in \mathrm{X}
\end{array}
$$

Function $g$ is the fitness function that reflects the total cost of a given permutation of gates. Commonly, it is used in the formulation that considers track minimization as primary objective and wire length minimization as a secondary one. Therefore, it is defined as $g\left(s_{k}\right)=\operatorname{I.J.TR}\left(s_{k}\right)+W L\left(s_{k}\right)$, or

$$
g\left(s_{k}\right)=I . J \cdot \max _{j \in\{1, \ldots, J\}} \sum_{i=1}^{I} b_{i j}+\sum_{j=1}^{J} \sum_{i=1}^{I} b_{i j}-I
$$

where the $I . J$ product is a weight to reinforce the part of the objective considering the maximum number of tracks and to make it proportional to the second part of the objective concerning the wire length. If $s_{k}$ is schema, the non-defined columns (\# label) are bypassed. It seems as these columns do not exist and the $b$ matrix used to compute $g\left(s_{k}\right)$ contains only columns with information. In the example of Fig. 3.c, the track number is $\max \{3, ?, 4, ?, ?, ?, 6,3, ?\}=6$ and the wire length is $\operatorname{sum}\{3+0+4+0+0+0+6+3+0\}-7=9$.

The other fitness function $f$ is defined to drive the evolutionary process to a population trained by a heuristic. The chosen heuristic is the 2-Opt neighborhood. Thus, function $f$ is defined by:

$$
f\left(s_{k}\right)=g\left(s_{v}\right), s_{v} \in\left\{s_{1}, s_{2}, \ldots, s_{V}\right\} \subseteq \varphi^{2-O p t}, g\left(s_{v}\right) \leq g\left(s_{k}\right)
$$

where $\quad \varphi^{2-o_{p t}}$ is a 2-Opt neighborhood of structure or schema $s_{k}$.

As the 2-Opt neighborhood can grow exponentially, in our implementation, only a fixed part, randomly chosen, of this space is used on each function call. Implementation details can be found in section III, subsection C. 
Considering the definition of $g$, the maximization objective on BOP appears to be a contradiction, but it is needed at the constructive phase that gives distinct treatments to structures and schemata. By definition, $f$ and $g$ is applied to structures and schemata, just differing in the amount of information and consequently in the values associated to them. More information means larger values. In this way, the $g$ maximization objective in $B O P$ drives the constructive phase of the $C G A$ aiming that schemata will be filled up to structures.

The optimal phase is conducted by the interval minimization $g-f$, which happens on structures near to a local 2-Opt minimum. It is interesting to note that a direct GMLP objective of tracks and wire length minimization is indirectly reproduced at the $(g-f)$ minimization.

\section{THE EVOLUTION PROCESS}

The $B O P$ defined above is not directly considered as the set $X$ is not completely known. Alternatively is considered an evolution process to attain the objectives (interval minimization and $g$ maximization) of the BOP. At the beginning of the process, two expected values are given to these objectives:

- $\quad$ maximization:

a non-negative real number $g_{\max }>\max _{s \in X} g(s)$ that is an upper bound on the objective value;

- interval minimization:

an interval length $d g_{\max }$, obtained from $g_{\max }$ considering a real number $0<d \leq 1$.

The evolution process is then conducted considering an adaptive rejection threshold, which contemplates both objectives in $B O P$. Given a parameter $\alpha \geq 0$, the expression

$$
g\left(s_{k}\right)-f\left(s_{k}\right) \geq d g_{\max }-\alpha \cdot d\left[g_{\max }-g\left(s_{k}\right)\right]
$$

presents a condition for rejection from the current population of a schema or structure $s_{k}$. The right hand side of (3.1) is the threshold, composed of the expected value to the interval minimization $d g_{\max }$, and the measure $\left[g_{\max }-g\left(s_{k}\right)\right]$, that shows the difference of $g\left(s_{k}\right)$ and $g_{\text {max }}$ evaluations.

Expression (3.1) can be examined varying the value of $\alpha$. For $\alpha=0$, both schemata and structures are evaluated by the difference $g-f$ (first objective of $B O P$ ). When $\alpha$ increases, schemata are most penalized than structures by the difference $g_{\max }-g$ (second objective of $B O P$ ). 
Parameter $\alpha$ is related to time in the evolution process. Considering that the good schemata need to be preserved for recombination, the evolution parameter $\alpha$ starts from 0 , and then increases slowly, in small time intervals, from generation to generation. The population at the evolution time $\alpha$, denoted by $P_{\alpha}$, is dynamic in size accordingly the value of the adaptive parameter $\alpha$, and can be emptied during the process. The parameter $\alpha$ is now isolated in expression (3.1), thus yielding the following expression and corresponding rank to $s_{k}$ :

$$
\alpha \geq \frac{d g_{\text {max }}-\left[g\left(s_{k}\right)-f\left(s_{k}\right)\right]}{d\left[g_{\text {max }}-g\left(s_{k}\right)\right]}=\delta\left(s_{k}\right) .
$$

At the time they are created, structures and/or schemata receive their corresponding rank value $\delta\left(s_{k}\right)$. These ranks are compared with the current evolution parameter $\alpha$. The higher the value of $\delta\left(s_{k}\right)$, and better is the structure or schema to the $B O P$, and they also have more surviving and recombination time.

For the $G M L P$, the overall bound $g_{\max }$ is obtained at the beginning of the $C G A$ application, by generating a random structure and making $g_{\max }$ receive the $g$ evaluation for that structure. In order to ensure that $g_{\max }$ is always an upper bound, after recombination, each new structure generated $s_{\text {new }}$ is rejected if $g_{\max } \leq g\left(s_{\text {new }}\right)$.

\section{A. Initial population}

The initial population is composed exclusively of schemata, considering that for each schema, a proportion of random positions receive a unique gate number. The remaining positions receive labels \#. Along the generations, the population increases by addition of new offspring generated out of the combination of two schemata.

\section{B. Selection}

There are two purposes on the evolution process: to obtain structures (good solutions to the $g$ maximization objective on the $B O P$ ), and that these structures be good ones (best solutions to the interval minimization objective on the $B O P$ ). The selection of structures and/or schemata for recombination will 
be conducted to attain these two objectives, selecting for recombination schemata with small number of labels \# and structures or schemata with small $d_{k}=\frac{g\left(s_{k}\right)-f\left(s_{k}\right)}{g\left(s_{k}\right)}$.

The structures and schemata in population $P_{\alpha}$ are maintained on ascending order, according to the key $\Delta\left(s_{k}\right)=\left(1+d_{k}\right) / \eta$, where $\eta$ is the number of genes containing information (not \#). Thus, good individuals with more genetic information (structures or semi-complete schemata) appear in first order places on the population.

Two structures and/or schemata are selected for recombination. The first is called the base $\left(s_{\text {base }}\right)$ and is randomly selected out of the first positions in $P_{\alpha}$, and in general it is a good structure or a good schema. The second structure or schema is called the guide $\left(s_{\text {guide }}\right)$ and is randomly selected out of the total population. The objective of the $s_{\text {guide }}$ selection is the conduction of a guided modification on $s_{\text {base }}$. Fig.5 represents the base-guide selection process.

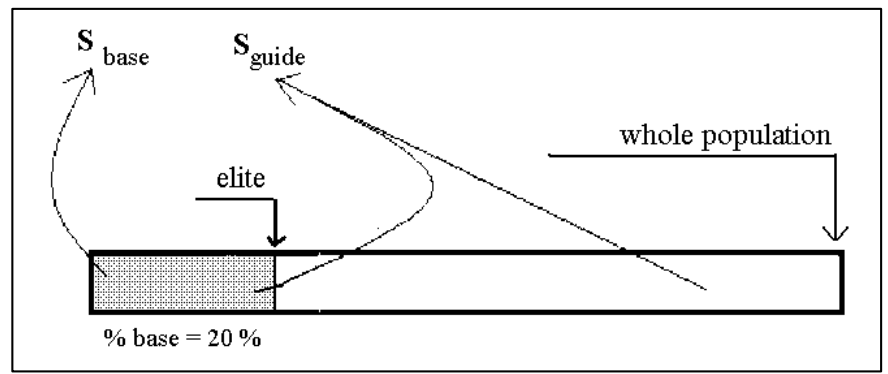

Fig.5 - Base-guide selection

\section{Recombination}

In the recombination operation, the current labels in corresponding positions are compared. Let $s_{\text {new }}$ be the new structure or schema (offspring) after recombination. Structure or schema $s_{\text {new }}$ is obtained by applying the following operations:

\section{\{ Recombination \}}

\section{For i from 1 to individual length}
I If $s_{\text {base }}$ (i) $=$ \#
and $s_{\text {guide }}(\mathrm{i})=\#$
II If $s_{\text {base }}$ (i) $<>\quad$ and $s_{\text {guide }}(\mathrm{i})=$ \#
III If $s_{\text {base }}$ (i) $=\# \quad$ and $s_{\text {guide }}($ i) $\langle>$ \#
IV If $s_{\text {base }}$ (i) $<>\# \quad$ and $s_{\text {guide }}(\mathrm{i}) \longleftrightarrow>$ \#

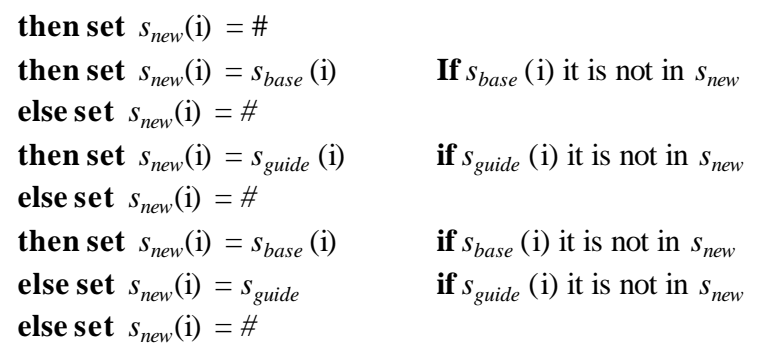


Observe that $s_{\text {base }}$ is a privileged individual to compose $s_{\text {new }}$, but it is not totally predominant. There is a small probability of the $s_{\text {guide }}$ gene information to be used instead of $s_{\text {base }}$ one.

\section{The algorithm}

The Constructive Genetic Algorithm can be summed up by the pseudo-code:

$C G A$ \{ Constructive Genetic Algorithm \}

Given $g_{\max }$ and $d ; \alpha:=0 ; \varepsilon:=0.005$

Initialize $\mathrm{P}_{\alpha}$;

For all $s_{k} \in \mathrm{P}_{\alpha}$ do compute $g\left(s_{k}\right), f\left(s_{k}\right), \delta\left(s_{k}\right)$

While (not stop condition) do

While (number of recombination) do

Select Base and Guide from $\mathrm{P}_{\alpha} ; \quad$ \{ selection operator \}

Recombine Base and Guide \{recombination operator \}

Evaluate Offspring;

Update Offspring in $\mathrm{P}_{\alpha \text {; }}$

end_while

$\alpha:=\alpha+\varepsilon$;

For all $s_{k} \in \mathrm{P}_{\alpha}$ satisfying $\alpha>\delta\left(s_{k}\right)$ do

end_while

end_for

Eliminate $s_{k}$ from $\mathrm{P}_{\alpha}$

The $\varepsilon$ increment is a linear step that increases the adaptive rejection threshold. Each distinct value of $\alpha$ corresponds to a generation. The stop conditions occur with an emptied population (assured by a sufficiently higher $\alpha$ ) or at a predefined number of generations. The population increases, after the initial generations, reaching an upper limit (in general controlled by storage conditions), and decreases for higher values of the evolution parameter $\alpha$.

The $C G A$ algorithm begins with the recombination procedures (over schemata only) and the constructive process builds structures (full individuals) progressively at each generation. The constructive process repeatedly uses genetic information contained in two individuals to generate another one. However, the constructive process can be complemented using especially designed mutation and filling heuristics, searching for a better overall performance. This filling process is called unary-filling operator and is applied before the recombination. It consists to fill the $s_{\text {base }}$ substituting the \# labels for gate numbers. For $G M L P$, this feature was implemented as following:
\{ initialization of parameters \}

\{ initial population \}

$\{$ fg-fitness and ranking \}

$\left\{\right.$ update $\left.P_{\alpha}\right\}$ 


\section{\{Unary-filling operator\}}

Select Base and Guide from $\mathrm{P}_{\alpha}$;

If Base is a schema then

Fill Base giving filled_Base;

end_if

Recombine Base and Guide

Mutation on full Base (or filled_Base);

Evaluate Offspring;

Update Offspring in $\mathrm{P}_{\alpha \text {; }}$

\author{
\{ selection operator \} \\ \{ heuristic for filling \} \\ \{ recombination operator \} \\ \{ local search on structures only \} \\ $\{$ fg-fitness and ranking \} \\ $\left\{\right.$ update $\left.P_{\alpha}\right\}$
}

This code fragment replaces the innermost while loop of the CGA pseudo-code. If the selected base is a schema, it is combined with the guide individual (schema or structure) giving a new individual. In any way, local search mutation is applied to a structure (full or filled base) and the offspring are updated depending on its rank. If a best structure is found, it is kept for the end.

For filling schemata it is used an algorithm based on neighborhood minimization. The principle is that a schema $s_{k}$ must be filled with a good sequence of gate columns that do not belong to it yet.

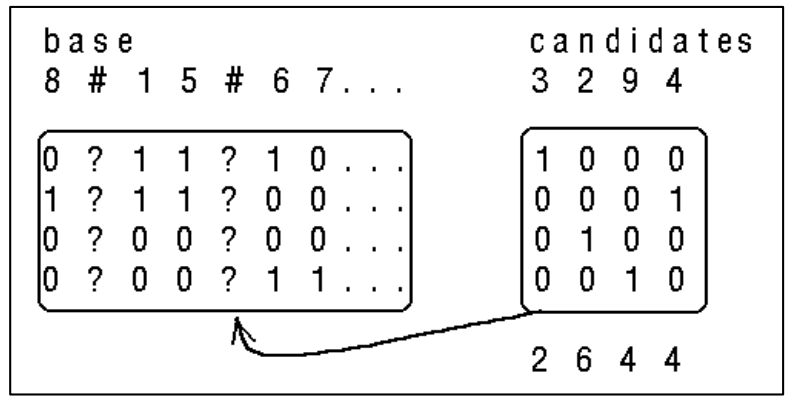

Fig.5 - Rule for filling schemata

In the example on Fig.6, there are 4 candidate gate columns (on right) to include in a partially showed schema at the marked position (\# position). For each \# position will be tested all candidate gate columns. It will be chosen the gate column that minimizes the bit-to-bit xor relation, considering, in matrix, the immediate neighbors gate columns to the left and to the right (if exists) of the \# point. The bit-to-bit xor sum of first candidate column is 2 , and is the smaller sum. This mechanism intends to decrease the possibility of zeros between ones at the included gate columns.

The local search mutation is always applied to structures, no matter how they are created (after recombination or after the filling process). The search at 2-Opt neighborhood of the structure (like the $f$ definition on expression 2.3) avoids that the computational effort increases defining a constant number of neighbors to be inspected until the best is found. The neighbors are generated by all the 2-move changes in a constant length part of the structure. A position is chosen at random and starting from there an iterative process that inspects all possible 2-move changes in the structure. 
The example of 2-move change is showed in Fig.7. The marks in positions of the structures mean reference points to be changed. Non-consecutive references cause the first change type, as showed in Fig.7a. Consecutive points cause the second change type in Fig. 7 b. Inspecting all or part of 2-Opt neighborhood needs several moves (catching 2-to-2 points). The number of 2-move changes (neighborhood width) on each local search mutation is a $C G A$ parameter of execution to be tuned. This and all the others settings will be described in next section.

The offspring is included in $\mathrm{P}_{\alpha}$ if its rank value $\delta$ is greater than the adaptive rejection threshold $\alpha$ and also it is not equal to any other individual in $\mathrm{P}_{\alpha}$.

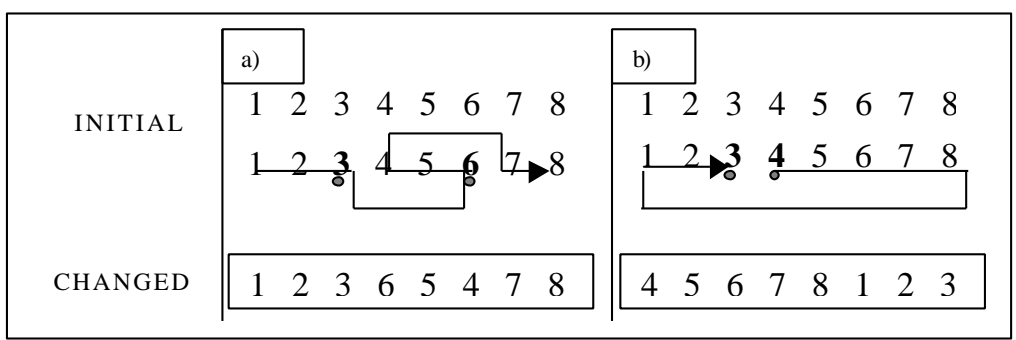

Fig.7 - Examples of one-move in 2-Opt neighborhood; a) non-consecutive reference points change; b) consecutive reference points change.

IV. COMPUTATIONAL RESULTS

The $C G A$ for $G M L P$ was coded in ANSI C and it was run on Intel Pentium II (266Mhz) hardware. For the computational tests, some $C G A$ parameters were adjusted. The $d$ parameter was set to 0.15 (usually between 0.10 and 0.20 values, for others applications [9]). This configures the interval $d . g_{\max }$, establishing the survival time of each individual, once the expected $\delta$ values are proportional to this interval. The $\varepsilon$ was set to 0.005 and also contributes to the survival time of each individual in $P_{\alpha}$. In this case, it is set to slow the increase on $\alpha$. These parameters avoid the premature termination with an empty population.

Each schema of the initial population received $50 \%$ of \# genes (indetermination percentage), and $10 \%$ up to $20 \%$ of population were considered base individuals for base-guide selection, determining a small degree of diversification in selection process. In this case, $10 \%$ up to $20 \%$ of better individuals of the population are considered for the selection of individuals base. 
Local search mutation rate was fixed in $100 \%$, which means a constant improvement of individuals. The number of individuals initially generated was proportional to problem length (at least the number of gates). Other important parameter to be tuned is the neighborhood width (nw) to each local search mutation. After some simulations the better results set $n w=20$. The ideal situation would be to use greater values for $n w$, but this would turn the mutation very slow.

The $C G A$ was applied to several instances taken from the literature. Previous tests showed that $C G A$ did not found any difficulties on small problems (smaller than v4090 problem), and the tests for some small problems are not reported in this work.

Table I presents the problem circuits considered, along with the corresponding number of nets and gates, a lower bound to the number of tracks, the previous best-known number of tracks (solution) and its reference. Chosen problems were the largest ones found in the literature and others for which authors have obtained recent improvements in results. The lower bound is computed by the maximum column sum of the original matrix. Most of the best previous results comes of Microcanonical Optimization $(M C O)$ approach [11], but other ones with same best results were included as in references: GM_Learn [12] and GM_Plan [13]. The $M C O$ overcame (or match) all the other approaches and it was chosen for comparison with the results of $C G A$.

\begin{tabular}{|cccccc|}
\hline problem & gate & net & LB & $\begin{array}{c}\text { best } \\
\text { tracks }\end{array}$ & Refs \\
\hline wli & 10 & 11 & 4 & 4 & {$[11,13]$} \\
wsn & 25 & 17 & 4 & 8 & {$[11,13]$} \\
v4000 & 17 & 10 & 5 & 5 & {$[11]$} \\
v4050 & 16 & 13 & 5 & 5 & {$[11,12]$} \\
v4090 & 27 & 23 & 9 & 10 & {$[11,12]$} \\
v4470 & 47 & 37 & 5 & 9 & {$[11,12]$} \\
x0 & 48 & 40 & 6 & 11 & {$[11,12]$} \\
w1 & 21 & 18 & 4 & 4 & {$[11,13]$} \\
w2 & 33 & 48 & 14 & 14 & {$[11,13]$} \\
w3 & 70 & 84 & 11 & 18 & {$[11]$} \\
w4 & 141 & 202 & 18 & 27 & {$[11]$} \\
\hline
\end{tabular}

Table I - Chosen problems to compare

Table II presents the comparison among the best results of $M C O$. The $M C O$ was run initially for 10 replications, but only after 1000 replications the authors reached their best results for problems wli, $v 4000, v 4470, x 0, w 3$ and $w 4$. The $C G A$ best results were obtained after 10 replications at same hardware environment. One replication means a complete execution over the same problem with the same 
parameters settings. For the $C G A$, the stop condition of one replication is when the best-known solution is reached or at a pre-defined number of generations with no improvement.

\begin{tabular}{|c|c|c|c|c|c|c|c|c|c|}
\hline \multirow[b]{2}{*}{ Prob } & \multicolumn{4}{|c|}{ MCO } & \multicolumn{5}{|c|}{ CGA } \\
\hline & $\begin{array}{r}\text { tracks:10 } \\
\text { replications }\end{array}$ & $\begin{array}{l}\text { tracks: } 1000 \\
\text { replications }\end{array}$ & $\begin{array}{r}\text { one trial } \\
\text { time } \\
\text { (avq) }\end{array}$ & $\begin{array}{r}1000 \text { trial } \\
\text { time }\end{array}$ & $\begin{array}{r}\text { Tracks:10 } \\
\text { replications }\end{array}$ & $\begin{array}{r}\text { wire } \\
\text { lenght }\end{array}$ & $\begin{array}{l}\text { Gen. } \\
\text { (avg) }\end{array}$ & $\begin{array}{r}\text { One trial } \\
\text { time } \\
\text { (avq) }\end{array}$ & $\begin{array}{r}10 \text { trial } \\
\text { time }\end{array}$ \\
\hline wli & 5.00 & 4.00 & 0.01 & 10.00 & 4.00 & 24 & 5.00 & 0.50 & 5.00 \\
\hline wsn & 8.00 & - & 0.01 & 10.00 & 8.00 & 98 & 7.00 & 1.50 & 15.00 \\
\hline v4050 & 5.00 & - & 0.01 & 10.00 & 5.00 & 41 & 5.00 & 0.50 & 5.00 \\
\hline v4000 & 6.00 & 5.00 & 0.01 & 10.00 & 5.00 & 53 & 5.00 & 0.50 & 5.00 \\
\hline v4470 & 10.00 & 9.00 & 0.70 & 700.00 & 9.00 & 246 & 33.00 & 66.50 & 665.00 \\
\hline v4090 & 10.00 & - & 0.10 & 100.00 & 10.00 & 95 & 13.50 & 2.03 & 20.33 \\
\hline$x 0$ & 11.00 & 11.00 & 0.70 & 700.00 & 11.00 & 303 & 92.57 & 75.56 & 755.60 \\
\hline w1 & 4.00 & - & 0.01 & 10.00 & 4.00 & 39 & 5.00 & 1.00 & 10.00 \\
\hline w2 & 14.00 & - & 0.40 & 400.00 & 14.00 & 235 & 19.50 & 18.50 & 185.00 \\
\hline w3 & 21.00 & 18.00 & 3.90 & 3900.00 & 18.00 & 677 & 186.00 & 306.25 & 3062.50 \\
\hline w4 & 32.00 & 27.00 & 61.70 & 61700.00 & 27.00 & 1730 & 225.00 & 5224.67 & 52246.67 \\
\hline
\end{tabular}

Table II - CGA comparison to MCO

To compare different number of replications with respect to execution time, the total time of all the replications was computed based on average of one trial execution time. Thus, the total time of $C G A$ experiments is 10 times the average of one trial execution. By its turn, the total time of $M C O$ experiments is 1000 times the average one trial execution. The "-" execution time, reported in [10] as close to zero, to our computations, was turned to 0.01 seconds, that represents the smallest fraction of one second with two decimal digits.

The "-" symbol in the "tracks: 1000 replications" column means that $M C O$ was not run 1000 times. According to the authors, for these problems, it was not necessary to improve the results obtained with 10 replications. The "gen" column is the number of generations (average) in 10 replications of $C G A$.

The numbers of tracks columns refer to the best result found for each problem. An additional $C G A$ column presents the wire length values, not reported in other references. Observing Table II, the CGA found the same results of $M C O$, but the total time of all replications to find the best results is smaller.

In [10], the authors remark the frequency of $36.3 \%$ to find the best result for wli problem (relatively small) in 1000 replications. No remarks are reported about larger problems. In our experiments, this percentage is $100 \%$ in 10 replications. In $C G A$, smaller percentages of best tracks were found only in larger problems (w3 and w4). There is no available data for a complete comparison of these two 
approaches, but, at least, $C G A$ seems to be more robust than $M C O$ in small problems, like the wli problem.

The Table III shows the CGA 5 best results (wire length and tracks) obtained in 10 replications. Problems $w 3, w 4, x 0 v 4470$ have less than $100 \%$ of frequency in reaching their best solution. The w4 problem appears to be the most difficult one, but these results show that no more than 10 trials were necessary to $C G A$ to find the best-known solution.

\begin{tabular}{|c|c|c|c|c|c|c|c|c|c|c|c|}
\hline & & & $C G A$ & $5 \mathrm{~b}$ & solut & ions & & & & & $\%$ best \\
\hline wsn & 104 & 8 & 104 & 8 & 105 & 8 & 107 & 8 & 113 & 8 & 100.00 \\
\hline wli & 18 & 4 & 18 & 4 & 18 & 4 & 18 & 4 & 18 & 4 & 100.00 \\
\hline v4050 & 41 & 5 & 41 & 5 & 41 & 5 & 42 & 5 & 42 & 5 & 100.00 \\
\hline v4000 & 53 & 5 & 53 & 5 & 53 & 5 & 54 & 5 & 55 & 5 & 100.00 \\
\hline v4470 & 246 & 9 & 253 & 9 & 265 & 9 & 266 & 9 & 282 & 9 & 100.00 \\
\hline v4090 & 95 & 10 & 96 & 10 & 96 & 10 & 98 & 10 & 99 & 10 & 90.00 \\
\hline$x 0$ & 303 & 11 & 304 & 11 & 305 & 11 & 306 & 11 & 308 & 11 & 80.00 \\
\hline w1 & 39 & 4 & 39 & 4 & 39 & 4 & 39 & 4 & 43 & 4 & 100.00 \\
\hline w2 & 235 & 14 & 236 & 14 & 267 & 14 & 273 & 14 & 275 & 14 & 100.00 \\
\hline w3 & 677 & 18 & 687 & 18 & 834 & 18 & 843 & 18 & 717 & 18 & 50.00 \\
\hline w4 & 1730 & 27 & 1836 & 27 & 1849 & 27 & 1745 & 28 & 1745 & 28 & 30.00 \\
\hline
\end{tabular}

Table III - Five best results and frequencies found for best tracks

\section{CONCLUSION}

This work describes an application of the Constructive Genetic Algorithm (CGA) to Gate Matrix Layout problems $(G M L P)$. The $C G A$ adapted to work with GMLP presents some new specific features, like the filling operator, and a 2-Opt heuristic used as mutation and on definition of the two fitness functions $(f$ and $g$ ). Regarding the computational tests, the $C G A$ reached all the best-known results (number of tracks) for instances taken from the literature, but it appears to be more robust than the Microcanonical Optimization approach. There is no published information about wire length in literature to completely compare the approaches. Applications of $C G A$ to other classes of permutation problems are foreseen for future works.

ACKNOWLEDGMENTS

The first author acknowledges the Programa Institucional de Capacitação Docente e Técnica PICDT/CAPES for financial support. The second author acknowledges Conselho Nacional de Desenvolvimento Científico e Tecnológico - CNPq (proc. 300837/89-5) and Fundação para o Amparo a 
Pesquisa no Estado de S. Paulo - FAPESP (proc. 99/06954-7) for partial financial support. The authors acknowledge the editors and the useful comments and suggestions of the three referees.

REFERENCES

1 Möhring, R. Graph problems related to gate matrix layout and PLA folding. Computing, Vol 7, pp. 17-51, 1990.

2 Kashiwabara, T. and Fujisawa T., NP-Completeness of the problem of finding a minimum clique number interval graph containing a given graph as a subgraph in Proc Symp. Circuits and Systems. 1979.

3 Goldberg, D.E., Genetic algorithms in search, optimization and machine learning. AddisonWesley, Reading, MA, p. 11-172, 1989.

Holland, J.H., Adaptation in natural and artificial systems. MIT Press, p. 11-147, 1975. Michalewicz, Z., Genetic Algorithms + Data Structures = Evolution Programs. SpringerVerlag, Berlin, 1996. Goldberg, D.E.; Korb, B.; Deb, K. Messy genetic algorithms: Motivation, analysis, and first results. Complex Systems v. 3: p. 493-530, 1989. Goldberg, D.E.; Deb, K.; Kargupta, H.; Harik, G. Rapid, accurate optimization of difficult problems using fast messy genetic algorithms. IlliGAL Report No. 93004, Illinois Genetic Algorithms Laboratory, Department of General Engineering, University of Illinois, Urbana, 1993.

8 Kargupta, H. Search, Polynomial Complexity, and The Fast Messy Genetic Algorithm, Ph.D. thesis, IlliGAL Report No. 95008, Illinois Genetic Algorithms Laboratory, Department of General Engineering, University of Illinois, Urbana, 1995.

9 Lorena, L. A. N. and Furtado, J. C. Constructive Genetic Algorithm for Clustering Problems. Evolutionary Computation 9(3): 309-327, 2001.

10 Ribeiro Filho, G. and Lorena, L. A. N. A Constructive Evolutionary Approach to School Timetabling. In Applications of Evolutionary Computing, Boers, E.J.W., Gottlieb, J., Lanzi, P.L., Smith, R.E., Cagnoni, S., Hart, E., Raidl, G.R., Tijink, H., (Eds.) - Springer Lecture Notes in Computer Science vol. 2037, pp. 130-139 - 2001 
11 Linhares, A., Yanasse H. and Torreão J. R. A. Linear Gate Assignment: a Fast Statistical Mechanics Approach. IEEE Trans. on Computer-Aided Designed of Integrated Circuits and Systems. Vol. 18(12), pp. 1750-1758. 1999.

12 Chen S. J. and Hu. Y. H. GM_Learn: an interactive learning algorithm for CMOS gate matrix layout. IEE Proc E, vol 137, pp 301-309, 1990.

$13 \mathrm{Hu}$ Y. H. and Chen S. J. GM_Plan: a gate matrix layout algorithm based on artificial intelligence planning techniques. IEEE Trans. Computer-Aided Designed, Vol. 9, pp. 836-845, 1990. 